\title{
Do dynamo-waves propagate along isorotation surfaces?
}

\author{
L. L. Kitchatinov ${ }^{\star}$ \\ Institute for Solar-Terrestrial Physics, PO Box 4026, Irkutsk 664033, Russia \\ Received 2 April 2002 / Accepted 20 June 2002

\begin{abstract}
It is shown that the basic rule of the dynamo theory on the propagation of dynamo-waves along surfaces of constant angular velocity is restricted to the case of isotropic magnetic diffusion. The influence of rotation on convective turbulence induces an anisotropy in the eddy diffusivity. The anisotropy makes the direction of travel of the dynamo wave deviate from the isorotation surfaces. The deviation is towards the equator for solar conditions. This finding may help to resolve the old problem of solar dynamo theory concerning the incompatibility between the helioseismological rotation law and equatorward migration of solar activity. A qualitative explanation for the inclined propagation is suggested. The derived properties of the dynamo waves are illustrated by 2D dynamo models in spherical geometry. The model with anisotropic diffusion shows a pronounced equatorial drift of toroidal fields even with a depth independent rotation.
\end{abstract}

Key words. Sun: magnetic fields - stars: magnetic fields - MHD

\section{Introduction}

Parker's (1955) dynamo-wave gives, perhaps, the clearest example of hydromagnetic dynamos. The example assumes that the typical scale of the magnetic field is small compared to the size of the body or the characteristic scale of its inhomogeneity. The short-wave approximation is normally violated in reality. Nevertheless, the results of global dynamo models that concern the bodies' geometry and do not assume the field scale to be small are readily interpreted in terms of dynamo-waves. Accordingly, studies of wave-type dynamos are still made extend. The WKB theory of stellar dynamo-waves travelling on a weakly inhomogeneous background (Galitsky \& Sokoloff 1999; Belvedere et al. 2000) and the theory of weakly nonlinear waves (Bassom et al. 1999) are among the most recent developments.

Concerning the Sun, the observed equatorward migration of magnetic activity over an 11-year cycle may be considered as a manifestation of the dynamo-wave's propagation (Parker 1979). The waves were found to propagate along lines of constant angular velocity (Yoshimura 1975). For this reason, the solar rotation was strongly believed to accelerate with depth until the internal rotation was detected by helioseismology. It is now evident that the angular velocity varies rather weakly with depth inside the Sun (cf., e.g., Shou et al. 1998). The helioseismological rotation law raises problems for the dynamo theory of solar activity (Parker 1987).

This paper aims to show that the problems can be at least partly resolved by allowance for anisotropy of solar convection. Yosimura's (1975) law holds for isotropic fluids. The rotational influence makes the convective cells elongate in the

\footnotetext{
^ e-mail: kit@iszf.irk.ru
}

direction of the rotation axis (Chandrasekhar 1961; Gillman \& Miller 1986). The effective diffusivity for the direction along the axis of rotation is larger than the diffusivity for the directions normal to the axis (Kitchatinov et al. 1994). We shall see that the anisotropy results in a deviation of the phase velocity of the dynamo wave from the isorotation surfaces. The deviation is not small and its direction is towards the equator for solar conditions. Note that the anisotropy results from the condition (rotation) that is vital for dynamos, which naturally leads to a consideration of dynamo-waves in anisotropic fluids.

The analytic analysis is made in Sect. 2. Section 3 illustrates the effect of anisotropy using 2D numerical simulations of dynamos in spherical geometry. We shall see that the butterfly diagram, computed with allowance for the anisotropy, shows an equatorial drift of the toroidal field even when rotation is uniform with radius.

\section{Dynamo-waves in anisotropic fluids}

We start with the induction equation for the mean magnetic field, $\boldsymbol{B}$, which allows for anisotropic turbulent diffusion

$$
\begin{aligned}
\frac{\partial \boldsymbol{B}}{\partial t} & =\operatorname{rot}(\boldsymbol{V} \times \boldsymbol{B}+\mathcal{E}), \\
\mathcal{E} & =\boldsymbol{\alpha} \circ \boldsymbol{B}-\eta \operatorname{rot} \boldsymbol{B}-\eta_{\|} \boldsymbol{e} \times((\boldsymbol{e} \cdot \nabla) \boldsymbol{B}) .
\end{aligned}
$$

In this equation, $\mathcal{E}$ is the so-called mean electromotive force (cf. Krause \& Rädler 1980), $\boldsymbol{V}$ is the fluid velocity, the $\boldsymbol{\alpha}$-tensor represents the alpha-effect by helical turbulence, $\eta$ is the isotropic part of the eddy diffusivity, and $\eta_{\|}$is the diffusivity excess for the preferred direction of anisotropy defined by the unit vector $\boldsymbol{e}$, i.e. total diffusivity for this direction equals $\eta+\eta_{\|}$. In the short-wave approximation, we treat all the coefficients of 
Eq. (1) as uniform except for the fluid velocity which possesses a uniform shear, $\boldsymbol{G}$,

$\boldsymbol{V}=\boldsymbol{j}(\boldsymbol{G} \cdot \boldsymbol{r}), \boldsymbol{j} \cdot \boldsymbol{G}=0$,

where $\boldsymbol{j}$ is the unit vector of the flow direction and $\boldsymbol{r}$ is the position vector.

Our model of a kinematic dynamo-wave roughly mimics the conditions in a stellar convection zone. The flow (2) corresponds to the stellar differential rotation and vector $\boldsymbol{j}$ points in the "azimuthal" direction. The mean magnetic field, $\boldsymbol{B}$, is assumed uniform along the $\boldsymbol{j}$-direction; this assumption corresponds to axial symmetry. The field can be decomposed into its toroidal $(B)$ and poloidal parts, the latter is defined by a toroidal potential $(A)$ :

$\boldsymbol{B}=\boldsymbol{j} B+\operatorname{rot}(\boldsymbol{j} A)$.

Apart from the eddy diffusivity, the alpha-effect may also involve anisotropy (Rüdiger 1990). This type of anisotropy is not relevant, however, to the $\alpha \Omega$-dynamo which is normally assumed to be at work in the Sun. The toroidal field generation by the alpha-effect is small for this type of dynamo so that only one (azimuthal) component,

$\alpha=\alpha_{m n} j_{m} j_{n}$,

of the $\alpha$-tensor remains important.

The linear dynamo problem for the homogeneous fluid reduces to the eigenvalue problem for the plane waves,

$B, A \sim \exp (\sigma t+\mathrm{i} \boldsymbol{k} \cdot \boldsymbol{r}), \quad \boldsymbol{k} \cdot \boldsymbol{j}=0$.

Substitution of (2)-(5) into (1) yields

$$
\begin{aligned}
\sigma B & =-\mathrm{i}((\boldsymbol{k} \times \boldsymbol{G}) \cdot \boldsymbol{j}) A-\left(\eta k^{2}+\eta_{\|}(\boldsymbol{k} \cdot \boldsymbol{e})^{2}\right) B, \\
\sigma A & =\alpha B-\left(\eta k^{2}+\eta_{\|}(\boldsymbol{k} \cdot \boldsymbol{e})^{2}\right) A .
\end{aligned}
$$

The compatibility condition for the equation system defines the eigenvalues. Those of them that may correspond to growing modes, $\mathfrak{R}(\sigma) \geq 0$, read

$$
\begin{aligned}
\sigma= & -\eta k^{2}-\eta_{\|}(\boldsymbol{k} \cdot \boldsymbol{e})^{2} \\
& + \begin{cases}\frac{1+\mathrm{i}}{\sqrt{2}} \sqrt{\alpha((\boldsymbol{G} \times \boldsymbol{k}) \cdot \boldsymbol{j})}, & \text { for } \alpha((\boldsymbol{G} \times \boldsymbol{k}) \cdot \boldsymbol{j})>0 \\
\frac{1-\mathrm{i}}{\sqrt{2}} \sqrt{-\alpha((\boldsymbol{G} \times \boldsymbol{k}) \cdot \boldsymbol{j})} & \text { for } \alpha((\boldsymbol{G} \times \boldsymbol{k}) \cdot \boldsymbol{j})<0 .\end{cases}
\end{aligned}
$$

The imaginary part of the eigenvalue, $\sigma=\gamma-i \omega$, give the wave frequency

$\omega=\mp \sqrt{\frac{1}{2}|\alpha((\boldsymbol{G} \times \boldsymbol{k}) \cdot \boldsymbol{j})|}$,

where the upper and lower signs correspond to the upper and lower lines in Eq. (7). The normalized increment, $\hat{\gamma}=\gamma /\left(\eta k^{2}\right)$, reads

$\hat{\gamma}=-1-a \cos ^{2}(\theta-\phi)+\sqrt{|\mathcal{D} \sin \phi|}$.

In this equation, $a=\eta_{\|} / \eta$ is the anisotropy parameter, $\theta$ and $\phi$ are the angles which the shear vector, $\boldsymbol{G}$, makes with the anisotropy direction, $\boldsymbol{e}$, and the wave vector, $\boldsymbol{k}$, respectively (note that $\theta$ is colatitude in the next section), and

$\mathcal{D}=\frac{\alpha G}{\eta^{2} k^{3}}$

is the dynamo number.

At this point, we have to decide whether the phase velocity or the group velocity of the dynamo-wave should be related to the observed latitudinal migration of solar activity. The group velocity is usually considered to be "more physical". This velocity with which the energy is transported is, indeed, of fundamental importance for wave excitations in ideal fluids that conserve their energy. The dynamo waves are quite different. They do not conserve their energy but exchange it with turbulent fluid. Their group velocity is of minor importance. What is meant by the latitudinal drift of solar activity is the change of the latitude of the largest rate of magnetic flux emergence with time. In other words, a hump of the wave is followed. This is the phase velocity

$\boldsymbol{v}_{\mathrm{p}}=\boldsymbol{k} \omega / k^{2}$.

The field amplification occurs when the dynamo number (10) exceeds a threshold value, $\mathcal{D}_{\text {cr }}$, where the increment $\hat{\gamma}$ (9) becomes positive. $\mathcal{D}_{\mathrm{cr}}=1$ for isotropic fluids with $a=0$. The strength of the rotational influence on convective turbulence is characterized by the Coriolis number,

$\Omega^{*}=2 \tau \Omega$,

where $\tau$ is the correlation time and $\Omega$ is the angular velocity. The representative value of the (depth dependent) solar Coriolis number is $\Omega^{*} \simeq 6$ (Durney \& Latour 1978). The quasilinear theory by Kitchatinov et al. (1994) gives the value of the anisotropy parameter, $a$, slightly below one for $\Omega^{*} \simeq 6$. We consider $a=1$ as a representative case.

Without loss of generality, the dynamo number is assumed positive (the change of sign reverses the directions of the wave vector and propagation velocity) and the orientation angles, $\theta$ and $\phi$, of the anisotropy and propagation direction are assumed to vary within the range from 0 to $\pi$. Figure 1 shows the threshold dynamo number and the angle $\phi$ at which it is achieved as functions of $\theta$.

The plots of Fig. 1 seem to have quite a clear meaning. When there is no anisotropy, $a=0$, the alpha-effect and diffusion do not depend on the direction of $\boldsymbol{k}$ but the fluid still possess a preferred direction $\boldsymbol{G}$. The flow is more efficient in shearing the toroidal field from the poloidal one the closer together the shear and the poloidal field directions are. Therefore, the maximum increment that defines $\mathcal{D}_{\mathrm{cr}}$ is achieved at the direction of $\boldsymbol{k}$ normal to $\boldsymbol{G}$. The wave propagates along the lines of constant (angular) velocity. With a finite anisotropy, $\mathcal{D}_{\mathrm{cr}}$ is larger compared to $\mathcal{D}_{\mathrm{cr}}=1$ for the isotropic case because of the additional diffusion, $\eta_{\|}$. The diffusive loss is smaller the closer to orthogonality the mutual orientation of vectors $\boldsymbol{k}$ and $\boldsymbol{e}$ is. When shear and anisotropy are parallel $\left(\theta=0^{\circ}, 180^{\circ}\right)$, the minimum diffusive loss and maximum shear efficiency occur at the same orientation of $\boldsymbol{k}$, which is still normal to the shear. When the shear and anisotropy are not parallel, however, the wave 

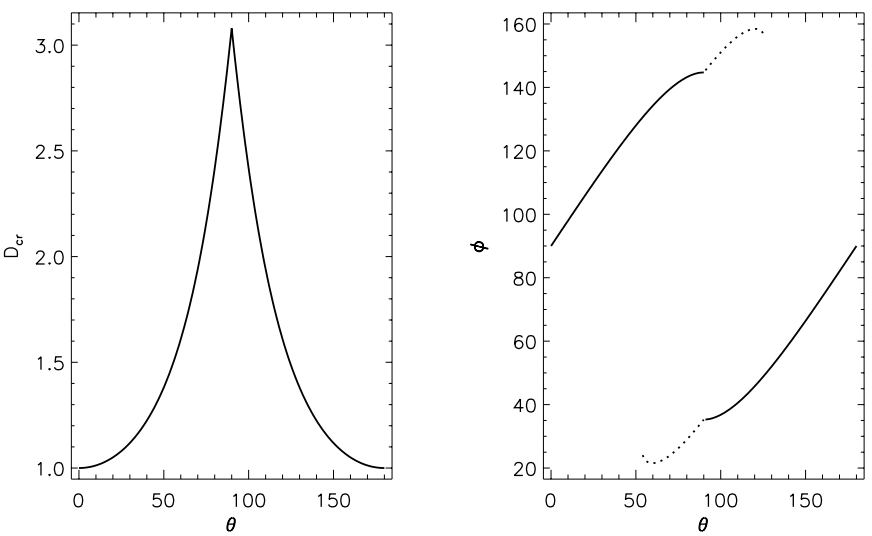

Fig. 1. Left: threshold dynamo number for field amplifucation as a function of the angle $\theta$ between shear and anisotropy directions. Right: the angle $\phi$ between the direction of the dynamo-wave propagation and the shear vector as a function of $\theta$. The dotted lines correspond to the secondary (negative) maximum in the dependence of the increment (9) on $\phi$. All angles are in degrees. The anisotropy parameter $a=1$.

vector declines from the line of constant velocity to decrease the diffusive loss. The phase velocity (11) turns in the direction that makes it closer to the orientation normal to the anisotropy direction. The velocity turns towards the equator for the solar case of (roughly) radial isorotation lines. The jump on the right side of Fig. 1 occurs because there are two maxima in the dependence of the increment (9) on $\phi$ in a range of $\theta$-values around $\theta=90^{\circ}$. The secondary (smaller) maximum is shown by the dotted line. Both maxima depend smoothly on $\theta$ but the primary (larger) one jumps from one branch of the dependence to another at $\theta=90^{\circ}$.

Note finally that the deviation angle of the dynamo wave propagation from the line of constant velocity is not small. The anisotropy has a considerable effect.

\section{2D models}

\subsection{Model design}

This section uses 2D dynamo models to illustrate the above findings for the effect of anisotropic diffusion. The models concern a magnetic field in a spherical layer representing the convection zone. The mean field is assumed symmetric about the axis of rotation.

The induction Eq. (1) for the toroidal field, $B$, and the potential, $A$, of the poloidal field now read

$$
\begin{aligned}
\frac{\partial B}{\partial t}= & \frac{\partial \Omega}{\partial r} \frac{\partial(\sin \theta A)}{\partial \theta}-\frac{\sin \theta}{r} \frac{\partial \Omega}{\partial \theta} \frac{\partial(r A)}{\partial r} \\
& +\frac{1}{r^{2}} \frac{\partial}{\partial r} \eta r^{2} \frac{\partial B}{\partial r}+\frac{1}{r^{2}} \frac{\partial}{\partial \theta} \frac{\eta}{\sin \theta} \frac{\partial}{\partial \theta} \sin \theta B \\
& +\left(\cos \theta \frac{\partial}{\partial r}-\frac{\sin \theta}{r} \frac{\partial}{\partial \theta}\right) \eta_{\|}\left(\cos \theta \frac{\partial}{\partial r}-\frac{\sin \theta}{r} \frac{\partial}{\partial \theta}\right) B, \\
\frac{\partial A}{\partial t}= & \alpha B+\frac{\eta}{r^{2}} \frac{\partial}{\partial r} r^{2} \frac{\partial A}{\partial r}+\frac{\eta}{r^{2}} \frac{\partial}{\partial \theta} \frac{1}{\sin \theta} \frac{\partial}{\partial \theta} \sin \theta A \\
& +\eta_{\|}\left(\cos \theta \frac{\partial}{\partial r}-\frac{\sin \theta}{r} \frac{\partial}{\partial \theta}\right)^{2} A,
\end{aligned}
$$

where $r$ is radius and $\theta$ from now on is colatitude.

For simplicity, the diffusivities, $\eta$ and $\eta_{\|}$, as well as the parameter $\alpha_{0}$ of Eq. (14) are assumed uniform. The simplest nonlinearity of alpha-effect quenching by the magnetic field is involved,

$\alpha=\alpha_{0} \cos \theta \Psi(\beta)$,

where $\beta=B / B_{\text {eq }}$ is the magnetic field normalized to the equipartition field which is also assumed constant with a plausible solar value of $B_{\text {eq }}=3000 \mathrm{G}$. The quenching function, $\Psi$, supplied by the quasilinear theory of the alpha-effect (Rüdiger \& Kitchatinov 1993) is adopted,

$\Psi(\beta)=\frac{15}{32 \beta^{4}}\left(1-\frac{4 \beta^{2}}{3\left(1+\beta^{2}\right)^{2}}-\frac{1-\beta^{2}}{\beta} \tan ^{-1} \beta\right)$.

The value $\eta_{0}=\eta+\eta_{\|} / 2=10^{12} \mathrm{~cm}^{2} \mathrm{~s}^{-1}$ for the mean diffusivity between the directions normal and parallel to the rotation axis is used.

The condition for the interface with a superconductor is employed at the inner boundary $r_{\mathrm{i}}=0.7 R_{\odot}$,

$\boldsymbol{E} \times \boldsymbol{n}=0$, at $r=r_{\mathrm{i}}$,

where $\mathcal{E}$ is the mean electromotive force of Eq. (1) and $\boldsymbol{n}$ is a unit vector normal to the boundary. No penetration of magnetic field from the radiative core is allowed $\left(A=0\right.$ at $\left.r_{\mathrm{i}}\right)$.

On the top boundary, the nonlinear condition for the toroidal field (Kitchatinov et al. 2000), which corresponds to the field escape through the Parker (1979) instability to the eruption of magnetic loops, and the condition for the poloidal field to be vertical are applied,

$\mathcal{E}_{\theta}+\frac{\eta_{0}}{R_{\odot}} \frac{B^{2}}{B_{0}}=0, \quad \frac{\partial(r A)}{\partial r}=0$ at $r=R_{\odot}$.

The magnitude of the threshold field for escape, $B_{0}=200 \mathrm{G}$, was chosen such that the amplitude of the surface toroidal field matches the observed strength (200-600 G) of the fields emerging in the solar active regions (Zwaan 1992; Lites et al. 1998).

Three models will be considered that differ in the anisotropy value and in rotation law. The models are specified in Table 1 . The effect of the anisotropy is most clearly pronounced with the rotation rate independent of radius. This type of rotation law is adopted in Models I and II. The dependence of the angular velocity on latitude is specified after the Doppler measurements by Howard \& Harvey (1970),

$\Omega=2.78\left(1-0.13 \cos ^{2} \theta-0.16 \cos ^{4} \theta\right) \mu \operatorname{rad~s}^{-1}$.

Model III employs the smoothed helioseismological rotation law by Belvedere et al. (2000). The model is merely aimed at showing that the equatorial drift of the toroidal field of Model II does not disappear with a realistic differential rotation. The two basic rotation laws are shown in Fig. 2.

Specification of the differential rotation, the diffusivity value, and geometry fully defines the parameter $C_{\Omega}=$ $\Delta \Omega R_{\odot}^{2} / \eta_{0}$ of the dynamo models. The value of the other key parameter, $C_{\alpha}=\alpha_{0} R_{\odot} / \eta_{0}$, remains uncertain because the value of $\alpha_{0}$ is indefinite. The $C_{\alpha}$ values used in the models are given 


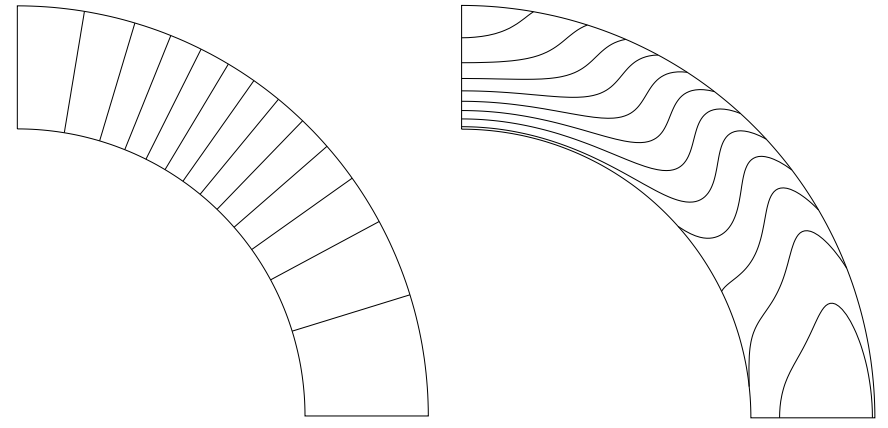

Fig. 2. Two rotation laws of the presented dynamo models. Left: depth-independent rotation of the Models I and II. Right: the smoothed helioseismological rotation law by Belvedere et al. (2000) used in the Model III.

Table 1. Specification of the dynamo models.

\begin{tabular}{cccc}
\hline \hline Model no. & Rotation law & Anisotropy, $a$ & $C_{\alpha}$ \\
\hline I & Radial & 0 & 2.96 \\
II & Radial & 1 & 2.76 \\
III & Helioseism. & 1 & 2.29 \\
\hline
\end{tabular}

in Table 1 . The values differ but they all are $10 \%$ above the corresponding threshold magnitudes for field generation. The solar dynamo is believed to be only slightly supercritical.

Global fields of the Sun are close to antisymmetry about the equator (Stenflo \& Güdel 1988). The dipole parity solutions are stable with Models II and III but not stable with the Model I. The dipole-type symmetry was imposed on Model I by the additional boundary condition at the equatorial plane,

$B=0, \partial A / \partial \theta=0$ at $\theta=\pi / 2$.

The dynamo Eqs. (13) were solved numerically by stepping them forward in time from a prescribed initial state. The solutions approached a steady cyclic behavior after several diffusive times. The results we are about to discuss all belong to these asymptotic oscillations.

\subsection{Results and discussion}

Figures 3 to 5 show the simulated butterfly diagrams for the radial field on the top boundary and toroidal field shortly below. The field evolution by the Model I is shown in Fig. 3. Only diffusive spreading of the magnetic pattern can be observed without any definite sense of migration. The model reproduces, in its main features, the old simulations by Köhler (1973).

Note that the cycle period is about three times shorter compared to the Sun. The simulated cycles can easily be made longer by decreasing diffusivity. There is no motivation for the tuning however. The adopted value of $\eta_{0}=10^{12} \mathrm{~cm}^{2} \mathrm{~s}^{-1}$ seems to be an underestimation already. Probably, the reason for the simulated cycles being too short is that the surface boundary condition (17) for the poloidal field is not realistic (Kitchatinov et al. 2000). Formulating an adequate condition for the poloidal field remains a problem. The problem is beyond the scope of this paper however. The same comment fully applies to the ratio
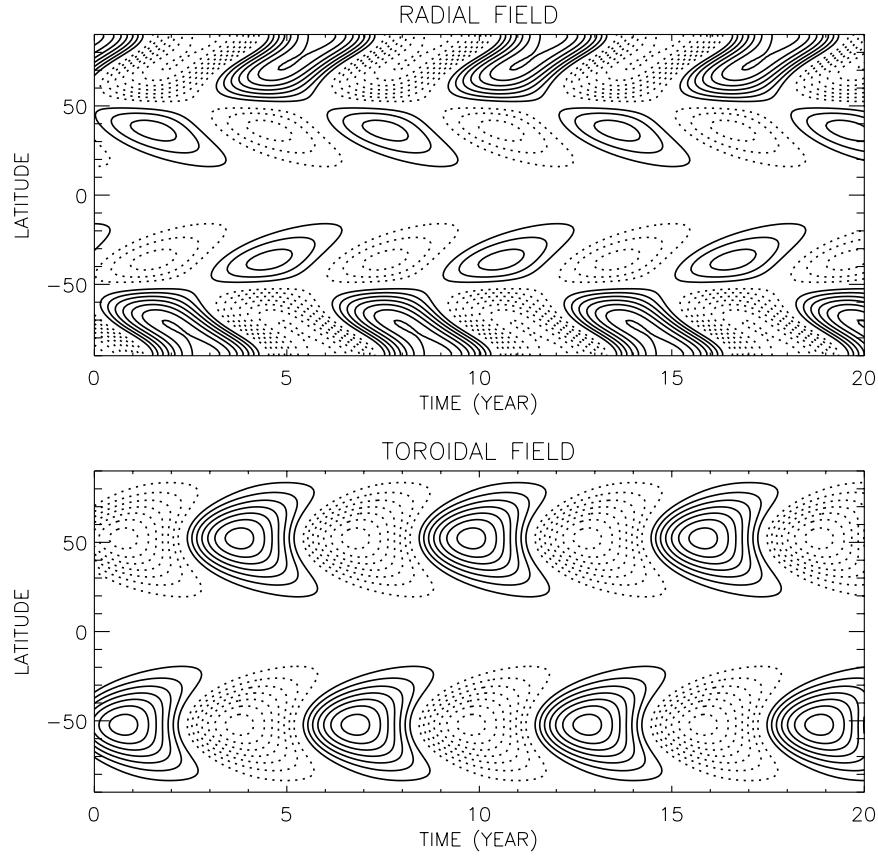

Fig. 3. Butterfly diagram for the depth-independent rotation (18) and isotropic diffusion (Model I). The isocontours for the radial field on the top boundary and the toroidal field shortly below are shown. Full and dashed lines show the positive and negative levels respectively. No latitudinal drift is found.
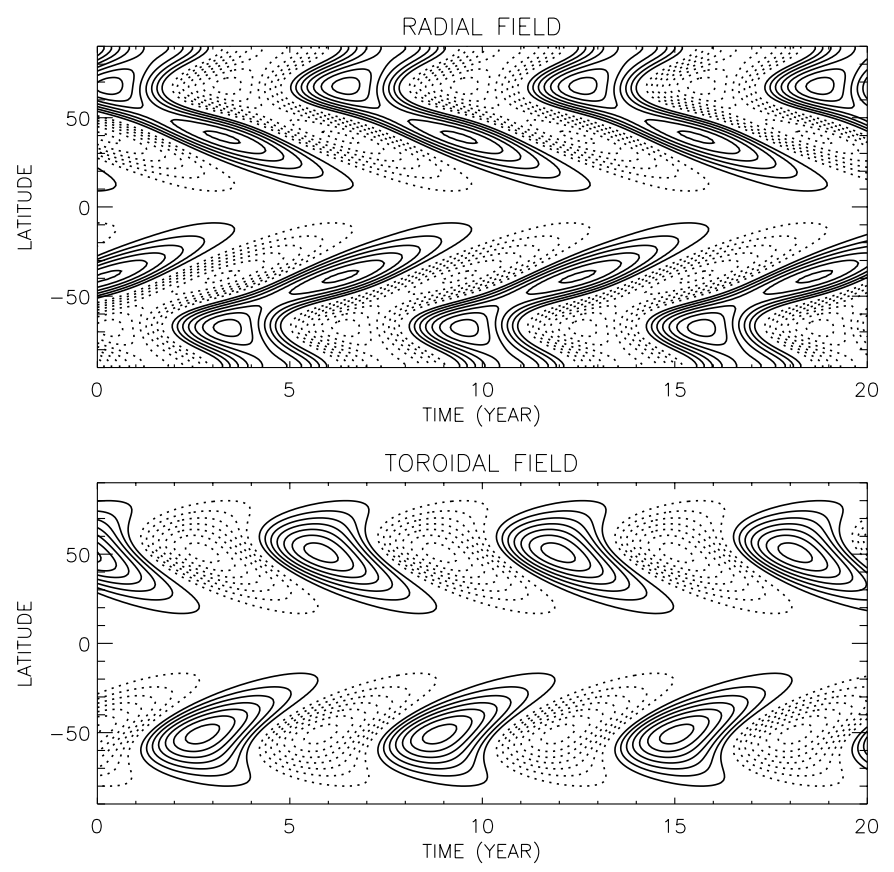

Fig. 4. Same as Fig. 3 but for Model II with anisotropic diffusion and depth-independent rotation. Equatorward drift of the toroidal field due to the diffusion anisotropy can be seen.

of toroidal to poloidal field which is typically around 20 with the present models, an order of magnitude smaller compared to the Sun. The ratio can be increased by decreasing diffusivity but such an "improvement" seems illusive. Anyway, the present simulations are aimed not at constructing a working model for the Sun but at illustrating the effect of anisotropy. 

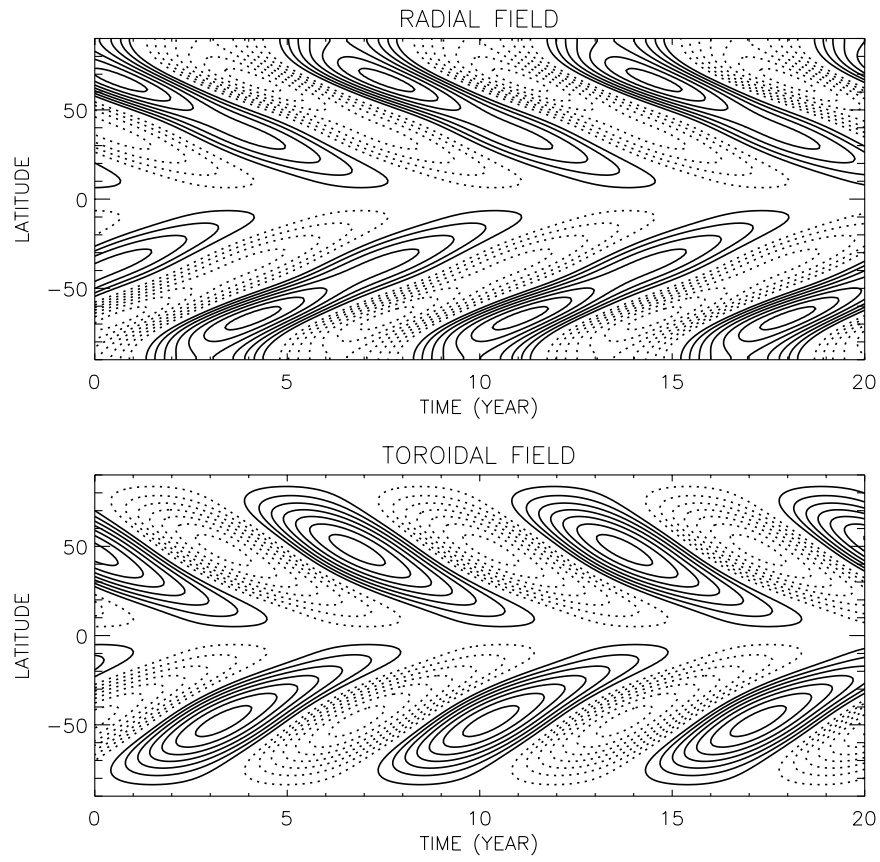

Fig. 5. Same as Fig. 3 but for Model III with smoothed helioseismological rotation and anisotropic diffusion.

As expected, an allowance for anisotropy of the eddy diffusion results in an equatorial drift of the magnetic field. The drift is well pronounced in Fig. 4. Note also that the poloidal field diagram of this figure is similar to the observational pattern (Stenflo 1988) and the solar-type phase relation, $B_{r} B_{\phi}<0$, is evident for a major portion of the plot, which is usual for the models with positive alpha-effect (Stix 1976). However, the toroidal fields of Fig. 4 extend to too high latitudes compared to the Sun.

Model III shows that the equatorial drift remains with the helioseismological differential rotation when the latitudinal migration is also influenced by radial inhomogeneity of the angular velocity (cf. Fig. 5).

Many other runs were made to check how sensitive the equatorial drift induced by the anisotropy is to details of the model. The runs confirm that it is not sensitive to boundary conditions or to the magnitude of the dynamo number.

Another possibility is that meridional circulation carries magnetic activity towards the equator (Dikpati \& Charbonneau 1999; Küker et al. 2001). The meridional flow can be efficient if the corresponding magnetic Reynolds number, $R m=v_{\mathrm{m}} R_{\odot} / \eta$, is sufficiently large. With the flow amplitude of some meters per second, the magnetic eddy diffusivity should be well below $10^{12} \mathrm{~cm}^{2} \mathrm{~s}^{-1}$. So low value of $\eta$ may be a consistent parameterization of convective turbulence only if the eddy diffusivity of magnetic field is much smaller than the eddy viscosity and/or thermal diffusivity. Otherwise, the fluid is still unstable to thermal convection (Tuominen et al. 1994). Theory of turbulent transport predicts, however, all the eddy diffusivities resulting from the same mixing being of the same order (Kitchatinov et al. 1994).
Note also that using anisotropic viscosity in dynamo models is not novel. Rüdiger et al. (1995) employed the rotationally induced anisotropy in their accretion disc dynamo. The equatorial drift due to the diffusion anisotropy was even obtained in the solar-type dynamo model (Kitchatinov et al. 1999) but not understood that time.

It can be concluded that accounting for the rotationally induced anisotropy of the eddy magnetic diffusivity may resolve the long-standing dilemma (Parker 1987) of the dynamo theory for solar activity.

Acknowledgements. This work was supported by INTAS under grant No. 2001-0550 and by the Russian Foundation for Basic Research (Project 02-02-16044).

\section{References}

Bassom, A. P., Kuzanyan, K. M., \& Soward, A. M. 1999, Proc. R. Soc. Lond. A, 455, 1443

Belvedere, G., Kuzanyan, K. M., \& Sokoloff, D. D. 2000, A\&A, 2000, 315,778

Chandrasekhar, S. 1961, Hydrodynamic and hydromagnetic stability (Clarendon Press, Oxford)

Dikpati, M., \& Charbonneau, P. 1999, ApJ, 518, 508

Durney, B. R., \& Latour, J. 1978, Geophys. Astrophys. Fluid Dyn., 9, 241

Galitsky, V. M., \& Sokoloff, D. D. 1999, Geophys. Astrophys. Fluid Dyn., 91, 147

Gillman, P. A., \& Miller, J. 1986, ApJS, 61, 585

Howard, R., \& Harvey, J. 1970, Sol. Phys., 12, 23

Kitchatinov, L. L., Pipin, V. V., \& Rüdiger, G. 1994, Astron. Nachr., 315,157

Kitchatinov, L. L., Pipin, V. V., Makarov, V. I., \& Tlatov, A. G. 1999, Sol. Phys., 189, 227

Kitchatinov, L. L., Mazur, M. V., \& Jardine, M. 2000, A\&A, 359, 531

Köhler, H. 1973, A\&A, 25, 467

Krause, F., \& Rädler, K.-H. 1980, Mean-field magnetohydrodynamics and dynamo theory (Akademieverlag, Berlin)

Küker, M., Rüdiger, G., \& Schultz, M. 2001, A\&A, 374, 301

Lites, B. W., Skumanich, A., \& Martinez Pillet, V. 1998, A\&A, 333, 1053

Parker, E. N. 1955, ApJ, 122, 293

Parker, E. N. 1979, Cosmical magnetic fields (Clarendon Press, Oxford)

Parker, E. N. 1987, Sol. Phys., 110, 11

Rüdiger, G. 1990, Geophys. Astrophys. Fluid Dyn., 50, 53

Rüdiger, G., \& Kitchatinov, L. L. 1993, A\&A, 269, 581

Rüdiger, G., Elstner, D., \& Stepinski, T. F. 1995, A\&A, 298, 934

Shou, J., Antia, H. M., Basu, S., et al. 1998, ApJ, 505, 390

Stenflo, J. O. 1988, Ap\&SS, 144, 321

Stenflo, J.O., \& Güdel, M. 1988, A\&A, 191, 137

Tuominen, I., Brandenburg, A., Moss, D., \& Rieutord, M. 1994 A\&A, 284,259

Stix, M. 1976, A\&A, 47, 243

Yoshimura, H. 1975, ApJ, 201, 740

Zwaan, C. 1992, The evolution of sunspots, in Sunspots: Theory and Observations, ed. J. H. Thomas, \& N. O. Weiss (Kluwer, Dordrecht), 75 